\title{
Immune Cell Profiles of Metastatic HER2 Positive Breast Cancer Patients According To The Sites of Metastasis
}

\section{Tiia J Honkanen}

Oulu University Hospital District: Pohjois-Pohjanmaan Sairaanhoitopiiri

\section{Milla E K Luukkainen}

Oulu University Hospital District: Pohjois-Pohjanmaan Sairaanhoitopiiri

\section{Antti Tikkanen}

Oulu University Hospital District: Pohjois-Pohjanmaan Sairaanhoitopiiri

\section{Peeter Karihtala}

Helsinki University Central Hospital Department of Oncology: Syopatautien klinikka

\section{Markus Mäkinen}

University of Oulu: Oulun Yliopisto

\section{Juha P Väyrynen}

University of Oulu: Oulun Yliopisto

Jussi Koivunen ( $\boldsymbol{D}$ jussi.koivunen@ppshp.fi )

Oulun Yliopisto https://orcid.org/0000-0001-6425-1640

\section{Research Article}

Keywords: Breast cancer, HER2, Liver metastasis, tumour profiles

Posted Date: May 13th, 2021

DOl: https://doi.org/10.21203/rs.3.rs-458719/v1

License: (c) (i) This work is licensed under a Creative Commons Attribution 4.0 International License. Read Full License

Version of Record: A version of this preprint was published at Breast Cancer Research and Treatment on November 24th, 2021. See the published version at https://doi.org/10.1007/s10549-021-06447-6. 


\section{Abstract}

Purpose: Recent works have characterized that metastatic site can affect the tumour immune profiles and efficiency of cancer immunotherapies. The prognosis of HER2 positive breast cancer is associated with the characteristics of the tumour immune microenvironment, with immunological cells playing a central role in efficiency of HER2 targeted antibodies. Here we investigated the prognostic significance of different metastatic sites and their correlation to tumour immune profiles in HER2 positive breast cancer treated with trastuzumab.

Methods: We collected all ( $n=54)$ HER2 positive metastatic breast cancer patients treated with trastuzumab containing regimens at Oulu University Hospital 2009-2014. Pathological and clinical data were collected from electronic patient records. The tumour immune profiles were analysed from pretreatment primary tumours using well-characterized immunological markers with computer-assisted immune cell counting.

Results: Of the metastatic sites, only liver metastases were associated with poor prognosis (hazard ratio $1.809,95 \%$ confidence interval $1.004-3.262$ ), especially when presenting as the primary site of metastases. Of the other sites, pulmonary metastases characterized a patient profile with trend to improved survival. Of the studied tumour immunological markers, liver metastases had low densities of $\mathrm{CD}^{+} \mathrm{T}$-cells $(\mathrm{p}=0.030)$ and $\mathrm{M} 1$-like macrophages in their primary tumours $(\mathrm{p}=0.025)$. Of the other studied markers and sites, pulmonary metastases had low STAB $1^{+}$immunosuppressive macrophage density in their primary tumours.

Conclusions: Our results suggest that the site of metastasis is associated with prognosis in HER2 positive breast cancer, highlighted by the poor prognosis of liver metastases. Furthermore, liver metastases were associated with adverse tumour immune cell profiles.

\section{Introduction}

Around $20 \%$ of all breast cancers have amplification of the human epidermal growth factor receptor 2 (HER2), which had been associated with aggressive tumour type and poor prognosis, before targeted therapies for HER2 were developed[1-4]. Immunology is suggested to play a major role in influencing the prognosis in HER2 positive breast cancer and treatment efficiency of HER2 targeted antibodies. The mode-of-action of HER2 antibodies is partly related to antibody-dependent cell cytotoxicity (ADCC) in which T-cells mediate the target cell killing[5, 6].

The appearance of distant metastases is associated with higher mortality in breast cancer[7]. In all subtypes of breast cancer, the most common sites of metastasis are bone, liver, lung, and brain. Bone metastases have often been linked to better prognosis, whereas patients with brain metastases are known to have the worst prognosis, especially in de novo stage IV breast cancers[8,9]. The metastatic pattern varies in different breast cancer subtypes; all subtypes are prone to metastasize to the bone, but brain and liver metastases are more often seen in HER2 positive and triple-negative breast cancers[8-12]. 
A recent study by Yu et al.[13] showed that liver metastasis can affect systemic anti-tumoural immunity by recruiting immunosuppressive macrophages into liver, which leads to deletion of cytotoxic T-cells and diminish the efficacy of immune checkpoint inhibitors. Our previous studies with metastatic HER2 positive breast cancer have demonstrated a strong association of cytotoxic T-cells and M1-like macrophages in primary tumours with better prognosis[14, 15]. Since immunology has a central role in HER2 positive breast cancer and targeted treatment acts through immune-mediated mechanisms[5], we speculate that the presence of liver metastases would accordingly be associated with adverse prognosis and specific tumour immune profiles of the primary tumour.

In the current study, we tested this hypothesis by evaluating the prognosis and tumour immune cell profiles of 54 patients with metastatic HER2 positive breast cancer treated with trastuzumab containing regimens.

\section{Results}

\section{Patients and Samples}

We searched pharmacy records of Oulu University Hospital and identified 54 patients who had received intravenous trastuzumab for metastatic breast cancer at least once during 2009-2014. Median age of the patients at the time of diagnosis was 58 years and 37 (68.5\%) of the patients were oestrogen receptor positive tumours (Table 1). Median overall survival and median survival in metastatic disease were 58 and 39 months, respectively. Of all the patients 22 (40.7\%) had primary metastatic disease at the time of diagnosis and 32 (59.3\%) had relapsed disease.

Table 1. Patient demographics $(n=54)$ 


\begin{tabular}{|ll|}
\hline & $\mathrm{n}(\%)$ \\
\hline Estrogen receptor positivity & $37(68.5)$ \\
\hline Primary metastatic disease & $22(40.7)$ \\
\hline Liver metastasis & $20(37.0)$ \\
\hline Primary liver metastasis & $12(22.2)$ \\
\hline Pulmonary metastasis & $20(37.0)$ \\
\hline Primary pulmonary metastasis & $17(31.5)$ \\
\hline Brain metastasis & $19(35.2)$ \\
\hline Primary brain metastasis & $2(3.7)$ \\
\hline Bone metastasis & $29(53.7)$ \\
\hline Primary bone metastasis & $25(46.3)$ \\
\hline Median age at diagnosis, years (range) & $58(28-82)$ \\
\hline Median survival, months (range) & $58(4-242)$ \\
\hline Median survival in metastatic disease, months (range) & $39(0-217)$ \\
\hline
\end{tabular}

The most common site of metastasis was bone, which was observed in 29 patients (53.7\%) and 25 of whom $(46.3 \%)$ had bone as a primary site of metastasis. Liver and pulmonary metastasis were both seen in 20 patients $(37.0 \%)$. Of the patients with liver metastasis, $12(22.2 \%)$ had liver as the primary site of metastasis, whereas $17(31.5 \%)$ of the patients with pulmonary metastasis had lungs as the primary site of metastasis. Brain metastasis was observed in 19 patients (35.2\%), but only $2(3.7 \%)$ had brain as the primary site of metastasis (Table 1 ).

In our previous studies with metastatic HER2 ${ }^{+}$breast cancer[14, 15], we found that high infiltration of both $\mathrm{CD}^{+}{ }^{+}$-cells and M1-like macrophages in the centre of the tumour (CT) were associated with improved survival. We updated the follow-up time and status of the patients and reanalysed the survival estimates. High density of $\mathrm{CD} 8^{+}$T-cells in CT (HR 0.321, 95\% $\left.\mathrm{Cl} 0.163-0.634\right)$, high density of M1-like macrophages in CT (HR 0.339, 95\% Cl 0.161-0.715) and the combination of CD8 ${ }^{+}$T-cells and M1-like macrophages in CT (high density of CD8 or M1 in CT; HR $0.246,95 \% \mathrm{Cl} 0.094-0.641$ ), and high density of $\mathrm{CD} 8$ and $\mathrm{M} 1$ in $\mathrm{CT}$ (HR $0.082,95 \% \mathrm{Cl} 0.025-0.272)$ predicted improved survival. With this updated follow-up, the survival differences were more pronounced than in our previous studies (Supplementary figure is provided in the Online Resource 1).

\section{Prognosis of the patients according to the metastatic sites}

Bone, liver, lungs, and brain were the most common sites of metastasis in this study, and these were selected for further analysis. The impact of the metastatic sites to the overall survival of the patients 
during the whole disease course were investigated with Kaplan-Meier analysis. Liver, bone, and brain metastases were linked with worse prognosis; however, only liver metastasis was statistically significant (HR 1.809, 95\% Cl 1.004-3.262, Fig. 1a).Interestingly, pulmonary metastasis showed tendency towards improved survival (HR $0.662,95 \% \mathrm{Cl} 0.361-1.216)$ but this, however, was not statistically significant (Fig. 1a).

Next, we evaluated whether the primary site of metastasis would also be linked to survival differences. We observed that liver (HR 4.171, 95\% Cl 2.009-8.656), bone (HR 2.047, 95\% Cl 1.115-3.757), and brain (HR 5.317, 95\% Cl 1.202-23.528) as a primary site of metastases were linked to poor prognosis, whereas primary pulmonary metastasis again showed tendency towards improved survival $(\mathrm{HR} 0.655,95 \% \mathrm{Cl}$ 0.346-1.239) (Fig. 1b).

\section{Correlation of the immune profiles and metastasis sites}

Next, we wanted to question, whether different metastatic sites were associated with variable primary tumour immune profiles. Since the survival of patients with liver and pulmonary metastasis varied most considerably, we chose these two sites for further analysis on immune profiles. Even though the sites of primary metastasis showed more significant survival differences, the number of primary cases was too low for reliable statistical analysis and, we therefore chose to analyze patients with either liver or pulmonary metastasis during the whole follow-up. We included numerous immunology related cell types (markers) in the analysis: T-cells (CD3), cytotoxic T-cells (CD8), regulatory T-cells (FoxP3), NK-cells (CD56), M1-like (iNOS) and M2-like (CD163) macrophages, immunosuppressive STAB1 ${ }^{+}$macrophages and IDO1 ${ }^{+}$ immune cells. We also evaluated IDO1 and CD47 expression in tumour cells. The scoring of the immunological cells was carried out using computer assisted counting of the positively stained cells $/ \mathrm{mm}^{2}$, while tumour cell markers were scored according to staining intensity or counted histoscore (based on the proportion of positive tumour cells and staining intensity).

T-cells and M2-like macrophages were the most abundant cell types both in the invasive margin (IM) and center of the tumour (CT) (Table 2). Mann-Whitney test was used to compare the median immune cell densities of the tumours, both in the IM and CT, in patient with or without liver and with or without pulmonary metastasis. Patients with liver metastasis had lower amount of $\mathrm{CD}^{+}{ }^{+}$-cells in the CT $(p=0.030)$ and M1-like macrophages in the CT $(p=0.025)$ than patients without liver metastasis (Fig. 2a). In addition, patients with pulmonary metastasis had lower amount of STAB1+ macrophages in the IM $(p=0.032)$ and CT ( $p=0.006)$ (Fig. $2 b)$. The other studied immunological markers did not show any statistically significant associations with liver or pulmonary metastasis (data not shown).

Table 2. Median densities of the studied immune cells 


\begin{tabular}{|c|c|c|c|}
\hline & $\begin{array}{l}\text { All cases, } \mathrm{n}=54 \text { (range) } \\
\left(\text { cells } / \mathrm{mm}^{2}\right)\end{array}$ & $\begin{array}{l}\text { Liver metastasis, } \mathrm{n}=20 \\
\text { (range) }\left(\text { cells } / \mathrm{mm}^{2}\right)\end{array}$ & $\begin{array}{l}\text { Pulmonary metastasis, } \mathrm{n}=20 \\
\text { (range) }\left(\text { cells } / \mathrm{mm}^{2} \text { ) }\right.\end{array}$ \\
\hline $\begin{array}{l}\text { CD3 } \\
\text { IM }\end{array}$ & $573(20-2520)$ & $479(71-1120)$ & $659(20-2459)$ \\
\hline $\begin{array}{l}\text { CD3 } \\
\text { CT }\end{array}$ & $242(19-2051)$ & $158(19-537)$ & $170(19-1641)$ \\
\hline $\begin{array}{l}\text { CD8 } \\
\text { IM }\end{array}$ & $207(20-1307)$ & $176(20-554)$ & $240(27-1307)$ \\
\hline $\begin{array}{l}\text { CD8 } \\
\text { CT }\end{array}$ & $120(4-1201)$ & $103(4-223)$ & $97(4-1201)$ \\
\hline $\begin{array}{l}\text { iNOS } \\
\text { IM }\end{array}$ & $42(4-145)$ & $32(4-78)$ & $38(10-99)$ \\
\hline $\begin{array}{l}\text { iNOS } \\
\text { CT }\end{array}$ & $37(4-173)$ & $20(4-53)$ & $36(6-172)$ \\
\hline $\begin{array}{l}\text { CD163 } \\
\text { IM }\end{array}$ & $338(24-957)$ & $421(219-653)$ & $372(24-653)$ \\
\hline $\begin{array}{l}\text { CD163 } \\
\text { CT }\end{array}$ & $295(15-983)$ & $267(37-695)$ & $321(15-678)$ \\
\hline $\begin{array}{l}\text { STAB1 } \\
\text { IM }\end{array}$ & $82(13-172)$ & $70(25-172)$ & $36(18-145)$ \\
\hline $\begin{array}{l}\text { STAB1 } \\
\text { CT }\end{array}$ & $30(7-118)$ & $33(12-72)$ & $18(7-36)$ \\
\hline
\end{tabular}

\section{Discussion}

Previous works have suggested that different anatomic locations of metastasis may correlate with tumour immunology. Especially liver metastasis has been linked to poor immunological tumour profiles and responses to immune checkpoint inhibitor treatments $[13,16]$. Tumour immunology has a central role in the prognosis of HER2 positive breast cancer and the cornerstone of its treatment are the HER2 targeted antibodies which effect significantly through immune activation[6, 17]. Therefore, we questioned whether the HER2 positive breast cancer with different metastatic sites would have altered survival and tumour immune profiles. We have previously shown that especially high number of CD8 positive T-cells and M1-like macrophages are associated with good prognosis in this disease[14, 15].

The association of different metastatic sites to survival has been extensively studied in breast cancers. Brain metastases have generally been associated with the poorest prognosis while bone metastases have been linked to better prognosis[8, 9, 18-20]. The association between metastatic sites and prognosis has less frequently been studied in HER2 positive breast cancer but there is some evidence suggesting similar survival tendencies as in other breast cancers[8]. Results of the current study suggest that liver metastases are associated with poorest prognosis while patients with pulmonary metastases 
showed the best prognosis. The adverse survival association was most significant, when patient presented with liver as the primary site of metastasis. Since our study included only patients who were treated with trastuzumab, the study might not capture patients with the poorest prognosis such as extensive brain metastasis who were never treated with systemic therapy. Our findings may suggest that the efficacy of HER2 antibody treatment might differ according to the metastatic site. Since liver metastasis are thought to induce general immunosuppression $[13,16]$ and HER2 antibodies mediate their effects through antibody-dependent cellular cytotoxicity (ADCC)[6, 17], immunosuppression might be account for the results. Importantly, however our study includes quite recent patients and the treatment paradigms of HER2 positive breast cancer have been evolving in the last years with introduction of new HER2 antibodies and antibody-linked cytotoxic payloads and older studies might not capture the effects of these treatments.

Tumour immune cell profiles have been widely studied in recent years. Especially high density of cytotoxic T-cells has been associated with good prognosis in numerous cancers[21-23]. We have previously characterized the immune cell profiles in HER2 positive breast cancer and have shown that especially, high densities of cytotoxic T-cells and M1-like macrophages in the center of the tumour region are strong independent prognostic markers surpassing all the studied baseline factors for survival[14, 15]. Here, we studied a large number of immunological markers in primary tumours and investigated their correlation to metastatic sites. As we hypothesized, we were able to detect differences in the immune microenvironment of the primary tumours according to the metastatic site. In brief, liver metastasis was associated with low densities of T-cells, and M1-like macrophages in the centre of the tumour, suggestive for an immunological desert or excluded type tumour microenvironment. Furthermore, pulmonary metastasis was associated with low STAB $1^{+}$macrophage density. STAB1 is an immunosuppressive marker associated with M2-like macrophages. One could speculate that altered tumour immunological microenvironment could account for the survival differences of the metastatic sites.

Our study has some weaknesses and strengths. The patient cohort was retrospectively collected, is limited in size, and adequate primary tumour samples were not available for all analyses. The lownumber of cases did not enable us to study tumour immune-profiles among the primary metastasis profiles which were the most prominently associated with prognosis. Therefore, the results should be verified in a larger, preferably prospectively collected, patient cohort. In the analysis for specific markers, we used only single, however well characterized, antibodies. We used computer-assisted cell counting which has been shown to surpass manual counting methods[24] and we analysed the markers in different tumour locations. Our patient cohort is extensively characterized and has a long follow-up and excellent outcomes with median survival of 58 months.

We envision that our study results could have potential clinical value. The study suggests that patient's prognosis in metastatic HER2 positive breast cancer can be defined by analysing the metastasis profiles. Furthermore, liver metastases define a patient group with poor prognosis and these patients also bare poor immunological features of the primary tumour. One could speculate that if the treatment results with the existing HER2 targeted therapies are ineffective with general immunosuppressive features of liver 
metastasis, better treatment outcomes could potentially be achieved with combinatory approaches or novel agents. Interestingly, previous works have characterized the role of M2-like macrophages in the liver metastasis-associated immunosuppression [13] and some M2-like targeting agents are under clinical evaluation [25]. Interestingly, our study showed an association between a low density of STAB1 positive cells, a marker for immunosuppressive macrophages, with good prognosis associated pulmonary metastasis. STAB1 targeting is also currently investigated in an early phase clinical cancer trial (NCT03733990).

In conclusion, the current study investigated prognosis of HER2 positive breast cancer according to metastasis sites and their associations with primary tumour immune profiles. We showed that liver metastasis is associated with poor prognosis and adverse tumour immune profiles. These results suggest that metastatic sites might characterize immunologically variable patient subgroups, and these could be used to stratify patients when investigating new treatment options.

\section{Materials And Methods}

\section{Patient data}

The patients for this retrospective study were identified through pharmacy records of Oulu University Hospital as previously described[26]. In short, all patient who had received intravenous trastuzumab for metastatic breast cancer and had adequate pre-chemotherapy samples available were selected for the study ( $n=54)$. HER2 positivity was determined by the presence of HER2 amplification in chromogenic in situ hybridization.

Most of the clinical data was gathered previously[14, 15, 26] but were updated for this study including the metastatic sites and the survival status in 01/2021. Overall survival was calculated from the time of diagnosis to death or end of follow-up. Survival in metastatic disease was calculated from the time of radiological or histological identification of metastatic disease to death or end of follow-up. Primary site of metastasis was defined as the site of metastasis at the time of diagnosis of metastatic disease.

\section{Immunohistochemistry and immune cell counting}

Immunohistochemical analyses of CD3, CD8, FoxP3, CD56, iNOS, CD163, ID01 and CD47 have been described in previous studies $[14,15]$. CD3, CD8 and CD56 staining were performed for 48 samples, FoxP3, iNOS, CD163, ID01 and CD47 for 40 samples and STAB1 for 26 samples due to a restriction of the tumour material. Immunohistochemical analysis of STAB1 was conducted on $3.5 \mu \mathrm{m}$ sections cut from paraffin-embedded specimens. The sections were first deparaffinized in HistoClear and rehydrated through graded alcohols. Antigen retrieval was performed with proteinase K (Agilent DAKO, \#S3020) for 5 minutes. Endogenous peroxidase activity was neutralized in $1 \% \mathrm{H}_{2} \mathrm{O}_{2}$-aqua solution for 20 minutes after which the samples were blocked using Vectastain ABC-HRP rat kit (Vector Laboratories, \#PK-6140). Primary antibody anti-STAB1 was a kind gift from Maija Hollmén (MediCity, University of Turku) and the samples were incubated with the antibody overnight $+4^{\circ} \mathrm{C}$. Bound antibodies were detected using the 
Vectastain ABC-HRP rat kit. Diaminobenzidine (DAB) was used as the chromogen and hematoxylin as the counterstain.

For the analysis of the immunohistochemistry, the tissue sections were scanned with Aperio AT2 imagecapturing device (Leica Biosystems). Imagescope (Aperio Technologies) software, version 11.2 was used to view the scanned images and capture 3-6 (median 6) images from the centre of the tumour (CT) and the invasive margin (IM) for immune cell counting. The criteria for the different tumour locations are previously described[14]. The cells were counted using earlier described and validated[24] computerassisted counting method, which utilizes ImageJ software.

\section{Statistics}

IBM SPSS Statistics 24.0 for Windows (IBM Corporation, Armonk, NY, USA) was used for statistical analyses. Survival analyses were performed with Kaplan-Meier method using log-rank test. Continuous variables between two groups were compared with Mann-Whitney -test and 2-tailed p-values were used. GraphPad Prism version 5 (GraphPad Software, San Diego, CA, USA) was used to create vertical scatter plots with median lines.

\section{Declarations}

\section{Funding}

This work was supported by the University of Oulu, Oulu University Hospital, and Finnish Cancer Institute.

\section{Conflicts of interest/Competing interests}

TJH, MEKL, AT, MM, JPV, PK declare no competing financial interests. JPK is a part-time employee of Faron Pharmaceuticals.

\section{Availability of data and material}

All data generated or analysed during this study are included in this published article. The raw data used in the analyses of the current study are not publicly available due to legislative issues concerning the privacy of the patients.

\section{Code availability}

Not applicable

\section{Author contributions}

JPK, TJH, MM, JPV and PK designed and coordinated the work. JPV, JPK, and TJH gathered data. JPK, TJH, AT and MEKL carried out statistical analysis. All the authors participated in analysis and interpretation of the data, and drafted, read, and approved the final version of the manuscript. 


\section{Ethics approval}

This study was performed under permits from National Supervisory Authority for Welfare and Health (9850/05.01.00.06/2010), the Northern Ostrobothnia Hospital District ethical committee (114/2011, amendment 23.02.2015) and the medical director of Oulu University Hospital (study no. 60/2015).

\section{Consent to participate}

At the time when the tumour samples were gathered, Finnish national legislation allowed the use of diagnostic tissues in scientific studies without an informed consent from the patient and therefore informed consents were not obtained. All the experiments were performed in accordance with relevant guidelines and regulations.

\section{Consent for publication}

All the authors' have read and approved the final version of the manuscript.

\section{Acknowledgements}

The authors express their gratitude to Ms Riitta Vuento and Ms Anne Ojala for their assistance in the preparation of the study material. This work was supported internally by the University of Oulu and Oulu University Hospital.

\section{References}

1. Slamon DJ, Clark GM, Wong SG et al (1987) Human breast cancer: Correlation of relapse and survival with amplification of the HER-2/neu oncogene. Science 235:182-191. https://doi.org/10.1126/science.3798106

2. Slamon DJ, Godolphin W, Jones LA et al (1989) Studies of the HER-2/neu proto-oncogene in human breast and ovarian cancer. Science 244:707-712. https://doi.org/10.1126/science.2470152

3. Marty M, Cognetti F, Maraninchi D et al (2005) Randomized phase II trial of the efficacy and safety of trastuzumab combined with docetaxel in patients with human epidermal growth factor receptor 2positive metastatic breast cancer administered as first-line treatment: The M77001 study group. J Clin Oncol 23:4265-4274. https://doi.org/10.1200/JC0.2005.04.173

4. Gonzalez-Angulo AM, Litton JK, Broglio KR et al (2009) High risk of recurrence for patients with breast cancer who have human epidermal growth factor receptor 2-positive, node-negative tumors 1 cm or smaller. J Clin Oncol 27:5700-5706. https://doi.org/10.1200/JC0.2009.23.2025

5. Arnould L, Gelly M, Penault-Llorca F et al (2006) Trastuzumab-based treatment of HER2-positive breast cancer: An antibody-dependent cellular cytotoxicity mechanism? Br J Cancer 94:259-267. https://doi.org/10.1038/sj.bjc.6602930

6. Gennari R, Menard S, Fagnoni F et al (2004) Pilot study of the mechanism of action of preoperative trastuzumab in patients with primary operable breast tumours overexpressing HER2. Clin Cancer Res 
10:5650-5655. https://doi.org/10.1158/1078-0432.CCR-04-0225

7. Chavez-MacGregor M, Mittendorf EA, Clarke CA et al (2017) Incorporating Tumor Characteristics to the American Joint Committee on Cancer Breast Cancer Staging System. Oncologist 22:1292-1300. https://doi.org/10.1634/theoncologist.2017-0116

8. Wang R, Zhu Y, Liu X et al (2019) The Clinicopathological features and survival outcomes of patients with different metastatic sites in stage IV breast cancer. BMC Cancer 19:1091.

https://doi.org/10.1186/s12885-019-6311-z

9. Wu SG, Li H, Tang LY et al (2017) The effect of distant metastases sites on survival in de novo stageIV breast cancer: A SEER database analysis. Tumor Biol 39:1010428317705082.

https://doi.org/10.1177/1010428317705082

10. Wu Q, Li J, Zhu S et al (2017) Breast cancer subtypes predict the preferential site of distant metastases: A SEER based study. Oncotarget 8:27990-27996.

https://doi.org/10.18632/oncotarget.15856

11. Kennecke H, Yerushalmi R, Woods R et al (2010) Metastatic behavior of breast cancer subtypes. J Clin Oncol 28:3271-3277. https://doi.org/10.1200/JC0.2009.25.9820

12. Xie J, Xu Z (2019) A population-based study on liver metastases in women with newly diagnosed breast cancer. Cancer Epidemiol Biomarkers Prev 28:283-292. https://doi.org/10.1158/10559965.EPI-18-0591

13. Yu J, Green MD, Li S et al (2021) Liver metastasis restrains immunotherapy efficacy via macrophagemediated T cell elimination. Nat Med 27:152-164. https://doi.org/10.1038/s41591-020-1131-x

14. Honkanen TJ, Moilanen T, Karihtala P et al (2017) Prognostic and predictive role of spatially positioned tumour infiltrating lymphocytes in metastatic HER2 positive breast cancer treated with trastuzumab.Sci Rep 7:18027. https://doi.org/10.1038/s41598-017-18266-1

15. Honkanen TJ, Tikkanen A, Karihtala P et al(2019) Prognostic and predictive role of tumourassociated macrophages in HER2 positive breast cancer. Sci Rep 9:10961. https: //doi.org/10.1038/ s41598-019-47375-2

16. Lee JC, Mehdizadeh S, Smith J et al (2020) Regulatory T cell control of systemic immunity and immunotherapy response in liver metastasis. Sci Immunol 5:eaba0759.

https://doi.org/10.1126/sciimmunol.aba0759

17. Hudis CA (2007) Trastuzumab - Mechanism of Action and Use in Clinical Practice. N Engl J Med 357:39-51. https://doi.org/10.1056/NEJMra043186

18. Arciero CA, Guo Y, Jiang R et al (2019) ER+/HER2 + Breast Cancer Has Different Metastatic Patterns and Better Survival Than ER-/HER2 + Breast Cancer. Clin Breast Cancer 19:236-245. https://doi.org/10.1016/j.clbc.2019.02.001

19. Kast K, Link T, Friedrich K et al (2015) Impact of breast cancer subtypes and patterns of metastasis on outcome. Breast Cancer Res Treat 150:. https://doi.org/10.1007/s10549-015-3341-3

20. Ording AG, Heide-Jørgensen U, Christiansen CF et al (2017) Site of metastasis and breast cancer mortality: a Danish nationwide registry-based cohort study. Clin Exp Metastasis 34:. 
https://doi.org/10.1007/s10585-016-9824-8

21. Ali HR, Provenzano E, Dawson SJ et al (2014) Association between CD8 + T-cell infiltration and breast cancer survival in 12439 patients. Ann Oncol 25:1536-1543. https://doi.org/10.1093/annonc/mdu191

22. Mahmoud SMA, Paish EC, Powe DG et al (2011) TumorTumour-infiltrating CD8 + lymphocytes predict clinical outcome in breast cancer. J Clin Oncol 29:1949-1955. https://doi.org/10.1200/JC0.2010.30.5037

23. Savas P, Virassamy B, Ye C et al (2018) Single-cell profiling of breast cancer T cells reveals a tissueresident memory subset associated with improved prognosis. Nat Med 24:986-993. https://doi.org/10.1038/s41591-018-0078-7

24. Väyrynen JP, Vornanen JO, Sajanti S et al (2012) An improved image analysis method for cell counting lends credibility to the prognostic significance of $T$ cells in colorectal cancer. Virchows Arch 460:455-465. https://doi.org/10.1007/s00428-012-1232-0

25. Liu Y, Wang R (2020) Immunotherapy Targeting Tumor-Associated Macrophages. Front Med 7:583708

26. Moilanen T, Mustanoja S, Karihtala P, Koivunen JP (2017) Retrospective analysis of HER2 therapy interruption in patients responding to the treatment in metastatic HER2 + breast cancer. ESMO Open 2:. https://doi.org/10.1136/esmoopen-2017-000202

\section{Figures}



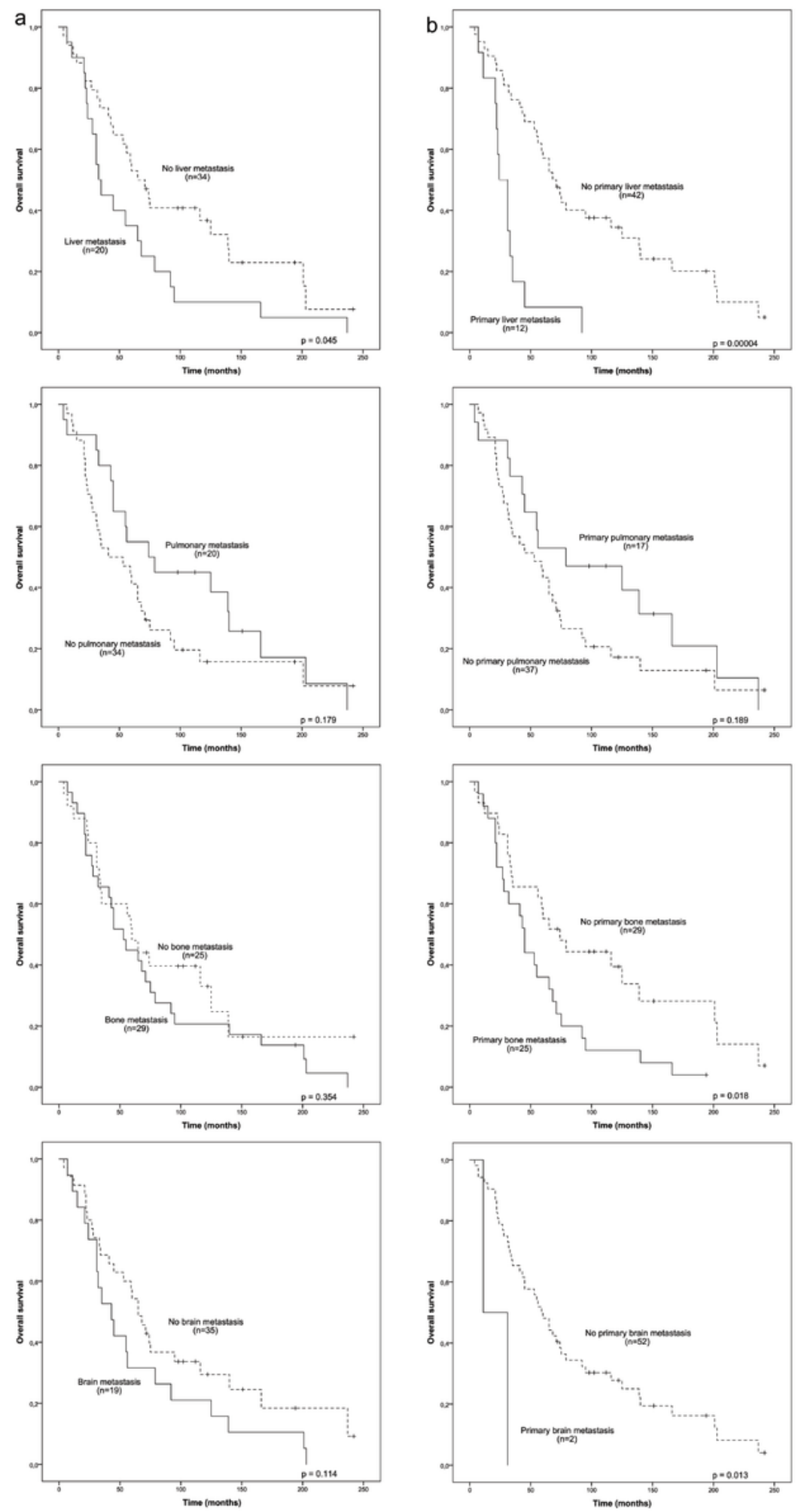

Figure 1

Survival analysis of metastatic HER2+ breast cancer patients according to the sites of metastasis. (A) Kaplan-Meier estimates demonstrate the difference in the overall survival of metastatic HER2+ breast cancer patients with or without liver, pulmonary, bone and brain metastasis. (B) Kaplan-Meier estimates illustrate the overall survival according to the first sites of metastasis. Crosses mark censored events. 

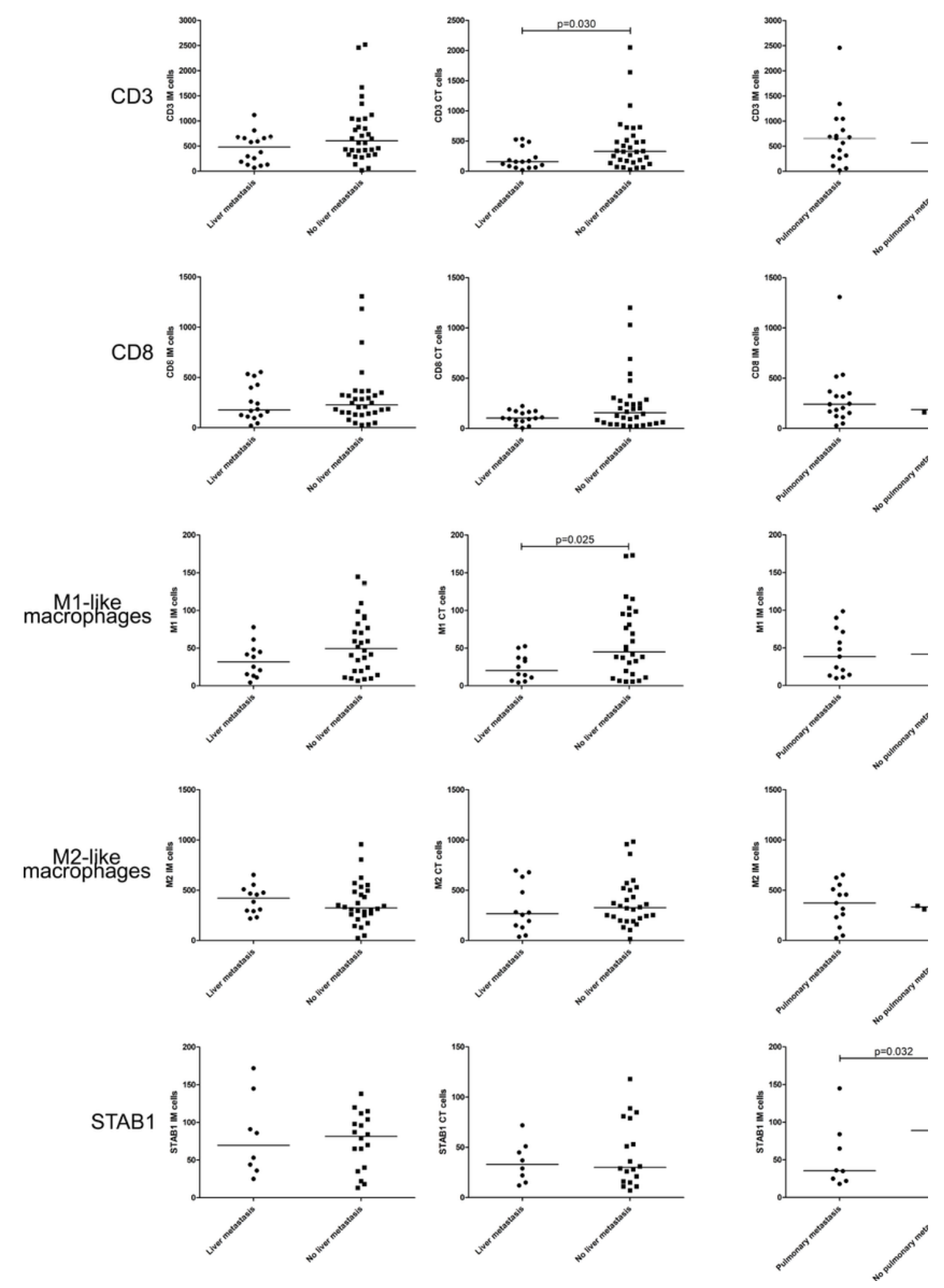

\section{Figure 2}

Immune cell densities in the invasive margin and center of the tumour in metastatic HER2+ patients with liver or pulmonary metastasis. Quantity of CD3, CD8, M1-like macrophages, M2-like macrophages and STAB1+ cells in the invasive margin and center of the tumour according to liver metastasis (A) or pulmonary metastasis (B) status. Median values for each immune cell types are marked as vertical lines. 
Mann-Whitney test was used to compare the median values of two groups, only significant $p$-values are presented.

\section{Supplementary Files}

This is a list of supplementary files associated with this preprint. Click to download.

- Supplementaryfig.1.pdf 